\title{
LAS INSTITUCIONES LOCALES DE DEMOCRACIA PARTICIPATIVA EN CÓRDOBA: UN ANÁLISIS DEL PERFIL DE SUS PARTICIPANTES
}

\author{
Patricia Mariel Sorribas ${ }^{1}$
}

\section{Introducción}

La afectación de la esfera gubernamental sobre casi todos los aspectos de la vida social es un hecho reconocido. Sin embargo, no implica cercanía ni congruencia entre ciudadanos y Estado. Este vínculo, que ha sido la base de la asociación entre Estado y democracia (Held, 1993) y que supone la existencia de una relación de simetria o congruencia entre los gobernantes y los destinarios de sus decisiones, ha sido puesta en duda por Lander (1998) al extenderla a la realidad de los Estados periféricos como los de la región. Esta duda cobra más sentido aún en relación a las innovaciones institucionales orientadas a propiciar la participación. Precisamente la justificación de la promoción de la participación ciudadana desde el Estado parte, en muchos casos, del propósito de acercar el Estado a los ciudadanos. Esta argumentación debe enmarcarse en una contextualización que ubica la distancia entre Estado y ciudadanos como parte del proceso histórico reciente en Argentina y en la región. Dicho proceso es demarcado por un orden neoliberal que supuso una reestructuración global del Estado en décadas pasadas, la subordinación de la política a la economía y una práctica política decisionista con su con-

\footnotetext{
${ }^{1}$ Centro de Investigación de la Facultad de Psicología (CIPsi), Universidad Nacional de Córdoba/Conicet y Facultad de Ciencia Política y Relaciones Internacionales, Universidad Católica de Córdoba. E-mail: patricia.sorribas@conicet. gov.ar.
} 
secuente consolidación de liderazgos personalistas (Svampa, 2005). Como hemos constatado, los procesos históricos de la región -y de Argentina en particular- han configurado distancias entre ciudadanos y Estados que más recientemente intentan ser acortadas mediante innovaciones en los diseños institucionales que propician una democracia más directa o participativa en el marco de una democracia representativa o indirecta (Eberhardt, 2010). El carácter novedoso de estas instituciones hace que su estudio a nivel poblacional esté aún inexplorado.

\section{Democratización de la democracia}

El marco conceptual de la democracia participativa permite entender algunas experiencias innovadoras -en términos de participación política institucional- como canales que encarnan la posibilidad de transformar el ejercicio de la ciudadanía y de redistribuir el poder a favor de la sociedad. Sin embargo, no están estas exentas de sufrir las consecuencias de prácticas perversas o intentos de cooptación. Precisamente porque las prácticas de democracia participativa "pretenden ampliar el canon político y, con eso, ampliar el espacio público y los debates y demandas sociales que lo constituyen, pueden ser cooptadas por intereses y actores hegemónicos para, en base a ellas, legitimar la exclusión social y la represión de la diferencia" (De Souza Santos, 2003:9). También en palabras de De Souza Santos (2003:9), cabe reconocer que "la perversión puede aparecer por muchas otras vías: por la burocratización de la participación, por la introducción de clientelismo bajo nuevas formas, por la instrumentalización partidaria, por la exclusión de intereses subordinados a través del silenciamiento o la manipulación de las instituciones participativas".

¿Por qué podrían estas experiencias transformar el ejercicio de la ciudadanía a nivel local? En principio, porque la apertura de nuevos espacios de participación supone cambios culturales, vinculados al inicio de procesos inclusivos 
y deliberativos. Luego porque en la apropiación por parte de los sujetos de estos nuevos modos de participación se re-significaría la noción de ciudadano, a partir de la concientización de la posesión de derechos (Goldfrank, 2007). Y también porque, al ser diseñadas dentro de procesos de descentralización del gobierno municipal atendiendo a territorialidades particulares, no partirían de suponer "un ciudadano abstracto sólo igualado por los derechos legales, sino un ciudadano con características específicas" (Rendón Corona, 2004: 186).

A su vez, democratizar la democracia alude a una democratización del Estado, a una construcción institucional que materialice una "nueva esfera pública autónoma, de carácter no estatal, situada en el espacio de tensión entre lo estatal y lo privado" (Rendón Corona, 2004: 194). La ampliación del espacio público transforma el ejercicio de la ciudadanía dotando a los ciudadanos de capacidades vinculadas a la "participación en la toma de decisiones, control sobre la gestión pública y participación en la ejecución de los planes decididos" (Rendón Corona, 2004: 195).

Tematizar la democratización de la democracia a su vez obliga a pensar y posicionarse respecto de la relación entre la democracia representativa y la democracia participativa. Esa relación puede ser significada como una de complementariedad, donde se presupone el reconocimiento por parte del gobierno de que el procedimiento participativo, las formas públicas de seguimiento de los gobiernos y los procesos de deliberación pública pueden sustituir parte del proceso de representación y deliberación que es concebido en el modelo hegemónico de democracia.

Para algunos autores, esa sustitución tendría posibilidades de realizarse si se lograra -mediante la creación de nuevas instituciones- la conformación de una esfera pública autónoma, de carácter no estatal, situada en el espacio de tensión entre lo estatal y lo privado. La nueva esfera debe ser capaz de llevar los intereses particulares al escenario público, donde serán confrontados con otros intere- 
ses privados y otros intereses de sentido universal (Utzig, 1998:113 citado en Rendón Corona, 2004).

Las posibilidades de confrontación de esos intereses particulares y más aún las que tengan los intereses de carácter universal revisten especial importancia al analizar innovaciones institucionales como las enmarcadas en la democracia participativa. Esto es así porque "la noción de soberanía del consumidor que elige entre opciones que difieren tan sólo en cómo lo afectan a él mismo, resulta imposible de trasladar al campo de la decisión política en la que el ciudadano debe elaborar su preferencia en torno a opciones que afectan también y de modo diferente a otros ciudadanos" (Maiz, 2001 :77).

En la literatura sobre estas temáticas es frecuente reconocer un supuesto sobre las motivaciones del ciudadano de a pie. Básicamente, se tiende a suponer que estas responden mayoritariamente a intereses particulares y, por ende, estos nuevos espacios de y para la política que propone la democracia participativa constituirian los escenarios propicios para transformarlos. Es decir, esas disposiciones de inicio pueden ser cambiadas al participar con otros de estos contextos específicos que implican tomar decisiones que afectan a otros, al menos a sus vecinos más inmediatos.

Son esos supuestos los que hacen proponer a Rendón Corona (2004) que para el funcionamiento de estas instituciones es necesario estimular un nuevo comportamiento político de las partes que no incluya las prácticas autoritarias y clientelistas por parte del Estado y los partidos, y los hábitos de confrontación e inmediatismo de la demanda económica por parte de las organizaciones comunitarias. Queda a la investigación empírica aportar evidencia sobre la emergencia de esos nuevos comportamientos. Y si estos tuvieran lugar queda por revelar en relación a qué tipo de decisiones políticas -que implican una distribución del poder- han emergido.

Esto último es de sumo valor si consideramos que, según los datos de algunos estudios, muchas de estas experien- 
cias no han democratizado las decisiones sobre orientaciones políticas; más bien se han concentrado en que los ciudadanos decidan sobre recursos materiales (Avritzer, 2002 citado en Rendón Corona, 2004).

Por otra parte, retomar el supuesto sobre las motivaciones y disposiciones del ciudadano cuando se involucra en política se hace necesario a fin de brindar evidencia que permita aportar datos relativos a poblaciones específicas y concretas, evitando así abstracciones genéricas sobre dicha población.

Entendemos que esto es posible asumiendo un enfoque psicosocial que permita conocer los perfiles de los ciudadanos locales y así entender luego sus prácticas políticas.

\section{La participación política en Córdoba: aproximaciones desde la psicología política}

A nivel nacional, desde el campo de la psicología política -considerado aún hoy como área emergente- la investigación sobre la participación política sigue siendo acotada y poco sistemática. En particular a nivel local su abordaje está en proceso de consolidación a través de diversos estudios desarrollados por el Equipo de Psicología Política (Facultad de Psicología - U.N.C.) desde 1997 (Brussino, Sorribas, Rabbia \& Imhoff, 2013). Mediante ellos se ha ido estableciendo la multidimensionalidad específica de la participación política de los cordobeses. Así, se pudo constatar que algunas de sus dimensiones se corresponden con las identificadas en otros estudios (Torcal, Montero y Teorell, 2006; Ferrer, Medina y Torcal, 2006). A su vez, en uno de los estudios poblacionales más recientes, Sorribas (2014) reportó 4 dimensiones participativas, correspondiendo 3 de ellas a dimensiones no electorales-de campaña: contacto con organizaciones y referentes políticos; acciones directas, de carácter contencioso, reivindicativas principalmente en el plano laboral-organiza- 
cional; y acciones de apoyo/rechazo al Poder Legislativo (nacional y provincial). Para esas 4 dimensiones también se han identificado sus variables explicativas (Sorribas, 2014; Sorribas \& Brussino, 2013a; Sorribas \& Brussino, 2013b).

Las instituciones locales propias de la democracia participativa como el Presupuesto Participativo y las Juntas de Participación Vecinal (JPV) constituyen una experiencia reciente de participación alentada desde el Estado municipal y pueden considerarse una nueva dimensión de la participación política institucional no electoral de la población local que ofrece un marco específico en términos espaciales y temporales. La participación en ellas resultó minoritaria entre la población de Córdoba y por ello no integran las 4 dimensiones participativas identificadas. Avanzar en su análisis se justifica, ya que alrededor de 2.600 cordobeses por año participan en ellas desde 2008. Adicionalmente, cabe destacar que quienes participaron de las JPV además reportaron participación en otras dimensiones (de contacto y contenciosa) (Sorribas \& Garay, 2014). La voluntad de poner en práctica estas modalidades participativas institucionalizadas obedece, además, a la "preexistencia de prácticas participativas de la sociedad" (Avritzer, 2002 en Rendón Corona, 2004: 189), hecho que se constata en el diseño de las JPV.

Si bien aún no se desarrolló un análisis explicativo específico de la participación política en estas instituciones, sí se han hecho avances descriptivos en el marco del análisis del repertorio político de los cordobeses (Garay, Ksiazenicki \& Sorribas, 2011; Red Ciudadana Nuestra Córdoba, 2013; Sorribas \& Garay, 2014) que permiten reconocer un bajo involucramiento con Centros Vecinales (15 \%), JPV (5\%) y Presupuesto Participativo (1\%). El análisis de la intensidad de la PP en el marco de estas y otras instituciones de democracia participativa permitió establecer que, en promedio, las valoraciones (afectividad/eficacia) han sido mayoritariamente altas (55 \%). 
Los indicadores de la Red Ciudadana Nuestra Córdoba (2011, 2012 y 2013) reportan variaciones en los niveles de participación en las JPV y el Presupuesto Participativo entre áreas de la ciudad y entre los períodos anuales de gestión. Estos resultados llevan a interrogarse sobre "los motivos o causas por las cuales se dan estas variaciones [las cuales] deberán ser objeto de análisis con los vecinos participantes, ya que ellos han sido y son los protagonistas de este espacio" (Red Ciudadana Nuestra Córdoba, 2011:11).

Considerando esta evidencia en esta primera aproximación podremos dar cuenta de un análisis comparativo entre participantes y no participantes de las JPV y el Presupuesto Participativo. Esto se justifica ya que la creación de estas nuevas instituciones puede hacer posible que el ciudadano tome parte en la definición e implementación de decisiones políticas (Garay Reyna, Ksiazenicki Viera \& Sorribas, 2011), ampliando así sus repertorios participativos. De este modo, podremos conocer qué características presentan los ciudadanos que efectivamente han ampliado sus repertorios dándoles cabida a estas instituciones de democracia participativa.

\section{Metodología}

La participación política analizada en el presente proyecto refiere a instituciones de Democracia Participativa implementadas en la ciudad de Córdoba. Por ello, procederé inicialmente en este apartado a describirlas sintéticamente.

Juntas de Participación Vecinal - Son un instituto público municipal de gestión asociada y articulada entre Centros de Participación Comunal (CPC) y Centros y Comisiones Vecinales, clubes, bibliotecas, centros de jubilados, escuelas, comedores, iglesias y otras entidades como las OSC, cooperadoras y otras instituciones barriales. Pueden participar en ellas vecinos que representen a esas organizaciones, mayores de 16 años y que residan o ejerzan actividad profesional/comercial dentro de la jurisdicción del CPC. Suponen la participación en partes iguales por género. Tienen por cometido diagnosticar 
diferentes tópicos urbanos, generar ideas y espacios de debate sobre proyectos y decisiones públicas de ámbito barrial (prioridades de obras, servicios y programas sociales para los barrios, ayuda para su ejecución).

Presupuesto Participativo - Es una forma de participación vecinal, voluntaria y universal, en la que la población debate, decide y controla el destino de los recursos a presupuestar para proyectos de obras, servicios y políticas sociales que realizará, prestará y ejecutará en el ejercicio siguiente la Municipalidad de Córdoba. Cada individuo puede involucrarse en calidad de vecino.

Muestra: se seleccionaron 3 CPC en base al nivel de participación en las JPV reportado entre 2008 y 2012: Villa Libertador, Empalme y Pueyrredón. Para cada CPC se seleccionaron dos zonas (A y B) donde se habian realizado actividades por el Presupuesto Participativo durante los dos últimos años.

Participantes: personas mayores de 16 años que habiten en los barrios de las zonas seleccionadas en el territorio de los 3 CPC elegidos.

Procedimiento: cuestionario administrado por el encuestador debidamente entrenado y previo consentimiento informado de la persona contactada en su domicilio o por otros medios (por teléfono o correo electrónico obtenidos de bases de datos provistas por la Red Ciudadana Nuestra Córdoba)

Instrumento: 1) sección de datos sociodemográficos tanto del encuestado como de su hogar; 2) sección de variables conceptualizadas como capital social por la literatura dedicada al análisis de la participación política considerando tanto su dimensión comportamental como la subjetiva; 3) sección con variables psicosociales ampliamente referenciadas como predictoras del involucramiento político en general y 4) sección para la escala de participación política (Sorribas, 2014).

Análisis de datos: Prueba T de Student para muestras independientes. 


\section{Resultados}

A continuación presentamos los resultados obtenidos mediante la Prueba $T$ de Student agrupados por set de variables que permiten diferenciar a los participantes de los no participantes en estas instituciones de democracia participativa. El primer set corresponde a las variables socio-demográficas analizadas. El segundo, a las variables psicosociales y el último, a variables comportamentales. Cabe aclarar que solo se presentan los datos de las variables para las que la diferencia de los grupos resultó significativa.

Luego en cada subsección correspondiente al set de variables se describen los resultados relativos a amplitud e intensidad de la participación política tanto en la muestra general ( $\mathrm{N}=267$ casos) como en la submuestra de participantes efectivos ( $\mathrm{N}=73$ casos).

\section{Variables sociodemográficas*}

Tabla 1

Diferencia de medias en variables sociodemográficas

\begin{tabular}{|l|c|c|c|c|}
\hline Variables & PP y/o JPV & N & Mean & Std. sd \\
\hline \multirow{2}{*}{ Edad del encuestado } & No PP & 195 & 39,32 & 15,944 \\
\cline { 2 - 5 } & Sí PP & 73 & 53,26 & 14,362 \\
\hline $\begin{array}{l}\text { Cantidad de personas } \\
\text { a cargo }\end{array}$ & No PP & 193 &, 96 & 1,288 \\
\cline { 2 - 5 } & Sí PP & 70 & 1,33 & 1,595 \\
\hline $\begin{array}{l}\text { Tiempo de residencia } \\
\text { del encuestado en el } \\
\text { barrio (años) }\end{array}$ & No PP & 188 & 24,1 & 16,128 \\
\cline { 2 - 5 } & Sí PP & 69 & 29,7 & 16,613 \\
\hline
\end{tabular}

* Solo se presentan los datos de las variables para las que la diferencia de los grupos resultó significativa.

A partir de los datos de la Tabla 1 podemos constatar que los ciudadanos que efectivamente participan en las JPV y/o en el PP tienen mayor edad, mayor cantidad de personas a cargo (contrario a lo que se supondría) y mayor tiempo de residencia en el barrio.

$\mathrm{Al}$ analizar la relación entre la amplitud de la participación de los encuestados y este set de variables se consta- 
tó solo una correlación positiva con la edad (.312 $p=.01$ ). $\mathrm{Y}$ al analizar la intensidad de la participación se observó una relación inversa con la edad $(-.147 p=.05)$ y con el tiempo de residencia del encuestado en el barrio (-.157 $p=.05)$. Luego, al revisar la relación entre amplitud de la PP y este set de variables socio-demográficas considerando la submuestra de participantes efectivos en JPV y/o Presupuesto Participativo, no se identificaron correlaciones. Y al analizar la intensidad de la PP se observó una correlación positiva con la cantidad de personas a cargo (.238 $p=.05) \mathrm{y}$ una correlación negativa con la edad (-.244 $p=.05)$

\section{Variables psicosociales*}

Tabla 2

Diferencia en medias de variables psicosociales

\begin{tabular}{|c|c|c|c|c|}
\hline Variables & PP y/o JPV & $\mathrm{N}$ & Mean & Std. sd \\
\hline \multirow{2}{*}{ Confianza Política (promedio) } & No PP & 195 & 3,24 & 668 \\
\hline & Sí PP & 73 & 3,54 & ,755 \\
\hline \multirow{2}{*}{ Normas de ciudadanía } & No PP & 195 & 16,54 & 4,153 \\
\hline & Sí PP & 73 & 19,56 & 3,768 \\
\hline \multirow{2}{*}{ Eficacia Política } & No PP & 195 & 17,90 & 5,483 \\
\hline & Sí PP & 73 & 21,84 & 4,986 \\
\hline \multirow{2}{*}{$\begin{array}{l}\text { Conocimiento Político (F1) } \\
\text { Cívico -Autoridades del PJ }\end{array}$} & No PP & 195 & 5,92 & 2,996 \\
\hline & Sí PP & 73 & 7,67 & 2,398 \\
\hline \multirow{2}{*}{$\begin{array}{l}\text { Conocimiento Político (F2) } \\
\text { Participativo-democracia participativa }\end{array}$} & No PP & 195 & 1,04 & 1,507 \\
\hline & Sí PP & 73 & 3,81 & 2,537 \\
\hline \multirow{2}{*}{$\begin{array}{l}\text { SDO: orientación hacia } \\
\text { la Igualdad Social }\end{array}$} & No PP & 194 & 17,35 & 2,845 \\
\hline & Sí PP & 73 & 18,33 & 2,000 \\
\hline \multirow{2}{*}{$\begin{array}{l}\text { SDO: orientación hacia } \\
\text { la Dominación Social }\end{array}$} & No PP & 194 & 9,32 & 4,035 \\
\hline & Sí PP & 73 & 7,93 & 4,665 \\
\hline \multirow{2}{*}{ Valor político: Colectivistas } & No PP & 195 & 25,14 & 4,834 \\
\hline & Sí PP & 73 & 27,18 & 2,715 \\
\hline \multirow{2}{*}{ Valor político: Individualistas } & No PP & 195 & 18,88 & 4,841 \\
\hline & Sí PP & 73 & 17,11 & 5,695 \\
\hline \multirow{2}{*}{ Interés Político } & No PP & 195 & 31,98 & 11,666 \\
\hline & Sí PP & 72 & 44,40 & 11,624 \\
\hline \multirow{2}{*}{$\begin{array}{l}\text { Atribuciones causales } \\
\text { de la PP: Internas }\end{array}$} & No PP & 194 & 17,70 & 4,414 \\
\hline & Sí PP & 73 & 19,40 & 3,483 \\
\hline
\end{tabular}

* Solo se presentan los datos de las variables para las que la diferencia de los grupos resultó significativa 
Los datos de la Tabla 2 nos permiten afirmar que quienes participan en Córdoba en estas instituciones de democracia participativa tienden a confiar más en instituciones y autoridades políticas, se guían más por las normas de ciudadanía, se perciben como más eficaces políticamente, cuentan con mayor información política, en términos ideológicos se orientan más hacia la igualdad social y menos hacia la dominancia social de un grupo sobre otro, se guían más por valores colectivistas y menos por valores individualistas, tienden a explicar la participación política por factores internos o disposicionales y expresan mucho mayor interés político.

Al analizar la relación de este set de variables con la amplitud de la PP se pudo evidenciar que correlaciona positivamente con: Normas de ciudadanía (.212 $\mathrm{p}=.01$ ), Eficacia Política Interna (.308 p=.01), Conocimiento Político Cívico (.322 $\mathrm{p}=.01)$, Conocimiento Político Participativo (.501 $\mathrm{p}=.01)$, Valores Políticos Colectivistas (.130 p=.05), Interés Político (.482 p=.01) y Atribuciones internas sobre la PP (.151 $\mathrm{p}=$.05) y negativamente con la Orientación hacia la Dominancia $(-.216 \mathrm{p}=.01)$ y con Valores Políticos Individualistas $(-.235 \mathrm{p}=.01)$.

En relación a la intensidad de la PP se pudo constatar que correlaciona positivamente con: Eficacia Política Interna (.142 $p=.05)$ y con Interés Político (.333 $p=.01)$. A su vez, correlaciona negativamente con: SDO Orientación a la Dominancia (-.224 p=.01) y Valores Políticos Individualistas $(-.286 \mathrm{p}=.01)$.

Al analizar las mismas relaciones considerando solo la submuestra de participantes en las JPV y/o Presupuesto Participativo se observó una correlación positiva entre la amplitud de la PP y el Conocimiento Político de tipo Participativo (.251 $p=.05)$ y una correlación negativa con el Valor Político Colectivista (-.333 $p=.01)$. A su vez, en relación a la intensidad de la PP se registraron correlaciones positivas con Eficacia Política Interna (.380 p=.01) y con Interés Político (.316 $p=.01)$ y correlaciones inversas con: SDO orienta- 
ción hacia la Dominación Social (-.271 $p=.05)$, Valor Político Individualista (-.271 $p=05)$ y Atribuciones causales Internas de la PP $(-.255 p=.05)$.

\section{Variables comportamentales}

Tabla 3

Diferencia en medias de variables comportamentales

\begin{tabular}{|l|c|c|c|c|}
\hline Variables & PP y/o JPV & N & Mean & Std. sd \\
\hline \multirow{2}{*}{ Amplitud de la PP } & No PP & 195 & 3,34 & 5,646 \\
\cline { 2 - 5 } & Si PP & 73 & 19,53 & 10,284 \\
\hline \multirow{2}{*}{ Intensidad de la PP } & No PP & 118 & 3,92 & 1,553 \\
\cline { 2 - 5 } & Si PP & 73 & 4,24 & 1,272 \\
\hline
\end{tabular}

Como puede reconocerse en los datos de la Tabla 3, quienes participan en las instituciones locales de democracia participativa participan más políticamente (amplitud) y con mayor sentido de eficacia y compromiso afectivo con las acciones que protagonizan (intensidad).

Por último, se analizó la relación entre amplitud e intensidad sin evidenciarse correlación alguna tanto en la muestra total como en la submuestra de participantes de las JPV y/o Presupuesto Participativo.

Para finalizar, se analizó ${ }^{2}$ el repertorio mayoritario de quienes efectivamente participaron o participan de las JPV y/o del Presupuesto Participativo $(N=73)$. De todos modos, se reportan las frecuencias y porcentajes de los principales formatos mediante los cuales estos ciudadanos se involucran en otras cuestiones políticas en la ciudad de Córdoba.

\footnotetext{
${ }^{2}$ No se pudo realizar un análisis factorial debido al bajo $\mathrm{N}$ de la submuestra.
} 


\section{Tabla 4}

Formatos y cantidad de participantes de las JPV y/o Presupuesto Participativo que los eligieron

\begin{tabular}{|c|c|}
\hline Formato & Participantes \\
\hline Contacto con Centro Vecinal & 66 \\
\hline Reclamo por iluminación pública & 60 \\
\hline Contacto con org. social o comunitaria & 55 \\
\hline Firma de petitorios & 54 \\
\hline Reclamos por atención primaria de la salud, transporte & 53 \\
\hline Hacer llegar ideas a concejales, intendentes. & 51 \\
\hline Contacto con org. civil & 48 \\
\hline Contacto con partido político & 48 \\
\hline Marchas & 47 \\
\hline Juntas de participación ciudadana $^{3}$ & 46 \\
\hline Fiscal de mesa para un partido & 45 \\
\hline Organizaste o recolectaste firmas para avalar una causa o petitorio & 44 \\
\hline Reclamos por basurales/inundaciones & 44 \\
\hline Se relacionó con medios masivos & 43 \\
\hline Contacto con punteros políticos & 40 \\
\hline Distribuir propaganda política & 40 \\
\hline Reuniones de equipos técnicos de partidos & 40 \\
\hline Audiencia pública & 38 \\
\hline Mítines partidarios & 38 \\
\hline Afiliación a partido político & 38 \\
\hline Contacto con gremio o sindicato & 37 \\
\hline Pegó afiches partidarios & 34 \\
\hline Paro/huelga & 33 \\
\hline Reclamos por el código de faltas & 27 \\
\hline Corte de calles o rutas & 26 \\
\hline Avales para partidos & 26 \\
\hline Acción por ley de medios & 23 \\
\hline Escraches & 22 \\
\hline Toma de edificio & 22 \\
\hline Sentadas & 21 \\
\hline Reuniones plan estratégico de la ciudad & 21 \\
\hline Acción por Ley de la COTBN & 18 \\
\hline Acción por resol. 125 & 15 \\
\hline Acción por la ley de matrimonio igualitario & 15 \\
\hline Acción por la revocatoria de mandato intendente & 15 \\
\hline
\end{tabular}

${ }^{3}$ Organizaciones avaladas en el marco de la Ley de Seguridad Pública 9235 de la provincia de Córdoba. 


\section{Discusiones}

En el presente trabajo nos propusimos aproximar una primera caracterización de los ciudadanos de Córdoba que participan en las JPV y/o en el Presupuesto Participativo. Constituye un primer paso en relación a la propuesta de desarrollar un modelo explicativo para esta dimensión no electoral de la PP institucional local.

En términos generales, podemos afirmar que un set de variables que ha demostrado su valor explicativo en relación a otras dimensiones de la participación política en Córdoba también se asocia diferencialmente a los perfiles de participantes-no participantes. Este hallazgo nos permite considerarlas como potenciales variables explicativas en futuros análisis que contemplen un mayor número de casos. A su vez, provee información respecto de los ciudadanos que están más cerca o más distantes de estas instituciones a pesar de haber sido diseñadas para canalizar la participación de todos los cordobeses mayores de 16 años.

Más especificamente, observamos que los estratos más jóvenes se mantienen más distantes de estas instituciones. En ambos grupos las medias de edad superan los 35 años; es más alta la media (superior a los 50 años) del grupo de participantes efectivos. Un análisis de proporciones permitió establecer que el género (F-M) no diferencia los grupos. Este dato es consistente con diferentes estudios locales (Brussino et al., 2013; Sorribas, 2014).

Como era de esperar, el tiempo de residencia en el barrio diferencia a los grupos. Este dato es consistente con el diseño mismo de las JPV, que prevé la pertenencia a organizaciones de diversa naturaleza como requisito para participar en ellas. A su vez, es consistente con el dato relativo a la edad promedio de los participantes efectivos.

Si bien se podría suponer que aquellas personas con mayores cargas familiares -en términos de comportamientos de cuidado y asistencia- tenderían menos a participar, encontramos la relación inversa. Este hallazgo requiere de un mayor análisis en futuras indagaciones. 
En este estudio por focalizarse en una modalidad de participación política institucional no electoral se incluyó la evaluación de las normas de ciudadanía. Se constató su aporte diferencial en relación a los dos grupos. Quienes participan expresan mayor sentido del deber respecto de determinados comportamientos del ciudadano. La percepción de las normas sociales de su contexto por parte de un individuo puede potencialmente afectar su comportamiento cívico. Por otra parte, las instituciones y los contextos son poderosos en la conformación de las normas sociales, por lo que el estudio de las normas sociales puede implicar la evaluación de los efectos institucionales de la participación, más que de la cultura cívica en sí misma (Jackman \& Miller, 2005). Tal interpretación resulta apropiada para los resultados reportados. Los cordobeses que participan consideran que es deber de un ciudadano ser activo y estar informado en politica (votar, formarse una opinión propia, realizar trabajo voluntario, ser parte de un jurado popular y participar de instituciones).

El sentido de eficacia interna que caracteriza a los participantes es consistente con una vasta literatura que aporta evidencia de la relación entre esta variable y distintas modalidades de participación política institucional (Imbrasaitè, 2009; Klesner, 2007; Mannarini, Legittimo \& Talò, 2008). De todos modos, y a diferencia de la dimensión contenciosa identificada a nivel local (Sorribas \& Brussino, 2013a), la eficacia política participativa (Wollman \& Stouder, 1991) no permitió diferenciar a los dos grupos. En nuestro trabajo la mayor especificidad situacional de los sentimientos de eficacia (relativos a los canales participativos) no se asoció a los participantes efectivos. En igual sentido se orientan los resultados respecto de la eficacia política colectiva. Por ello, no pudimos constatar la relación reportada por otros autores. En Córdoba, la sensación de ser capaz de lograr un objetivo mediante la realización de acciones colectivas no resultó ser más poderosa que la conciencia acerca del vínculo establecido entre habilida- 
des personales de uno mismo y los resultados de la acción (Mannarini et al., 2008).

En consistencia con el resultado del sentido de eficacia política interno (mayor en participantes efectivos), las atribuciones causales sobre la participación política de los ciudadanos que se vincularon a las JPV y/o el Presupuesto Participativo correspondieron a un locus de control interno. Este hallazgo es consistente con los reportados a nivel local en relación a la dimensión electoral y la dimensión de influencia sobre el Poder Legislativo (Sorribas \& Brussino, 2013b). Demuestra así ser una variable relevante para entender la participación institucional.

Es de destacar la diferenciación establecida por la SDO. La teoría de la dominancia social propone que los grupos e instituciones promueven cogniciones que refuerzan o bien atenúan la desigualdad grupal (Van Laar \& Sidanius, 2001). Estas cogniciones o ideologías son denominadas mitos legitimadores y algunos se orientan a alentar la jerarquía legitimando la desigualdad grupal y otros se orientan a atenuar la jerarquía legitimando la búsqueda de la igualdad grupal (Guimond, Dambrun, Michinov \& Duarte, 2003). Según nuestros resultados, los participantes se caracterizan por una mayor tendencia a no legitimar la desigualdad grupal y a buscar la igualdad grupal. En base a ello cabe preguntarse si estas instituciones de democracia participativa son percibidas como oportunidades para realizar esas tendencias ideológicas en términos de toma de decisiones que afectan a los ciudadanos más cercanos territorialmente.

Los valores políticos colectivistas también permitieron diferenciar a ambos grupos. Este hallazgo es consistente con los resultados obtenidos para otras dimensiones de la participación local: contenciosa, electoral y de influencia sobre el Poder Legislativo (Sorribas \& Brussino, 2013a y 2013b). El carácter egoísta o autointeresado de los valores políticos individualistas se asocia más a los no participantes en estas instituciones. 
El hecho de que los participantes efectivos se muestren más confiados respecto de instituciones y autoridades es esperable. Esta variable en estudios previos no demostró valor explicativo respecto de otras dimensiones de la participación local. Por ello habría que profundizar el análisis sobre la relación hallada desagregando los ítems específicos que conforman la escala.

Por último, las JPV y el Presupuesto Participativo resultan opciones de participación para ciudadanos que demuestran una alta participación (amplitud) mediante otros formatos y un alto compromiso con esas acciones (intensidad). Es decir, amplian la oferta de participación a quienes ya se mostraban movilizados políticamente. Estos se acercan más a estas instituciones. Estos resultados justifican un análisis con mayor detalle sobre estas variables comportamentales junto con la pertenencia a redes sociales en el territorio (dimensiones del capital social). En tal sentido se orientan los trabajos en curso del equipo.

Considerando el análisis sobre las prácticas o formatos mayoritarios de los participantes efectivos podemos reconocer la importancia que reviste el actor partido politico en el marco de una participación institucional no electoral como la estudiada aquí. Es de notar tanto la cantidad de los formatos (9) como la de participantes efectivos que reconocieron emplearlos (entre 48 y 26 participantes). Este hallazgo justifica una indagación más detallada de esta relación en términos de preferencias partidarias y a su vez del rol de delegado cumplido por los vecinos en el marco de las JPV. Queda también por establecer si esta cercanía mayoritaria con partidos políticos modela tanto la intensidad de la participación política como el sentido de eficacia percibido por los participantes efectivos.

El carácter minoritario de los formatos orientados a influenciar decisiones del Poder Legislativo (nacional o provincial) resulta contradictorio con datos obtenidos en población general en estudios previos (Sorribas, 2014). Que quienes participan o participaron efectivamente en las JPV 
y/o Presupuesto Participativo reporten este dato puede obedecer al carácter más local de su involucramiento político, sobre cuestiones materiales que afectan a las condiciones de vida de los vecinos de barrios específicos de la ciudad de Córdoba. Es decir, correspondería más a un perfil de "un ciudadano con características especificas" (Rendón Corona, 2004: 186) que atiende a territorialidades particulares.

Por último, en términos de factores predictores de la participación política institucional no electoral, debemos reconocer que el análisis correlacional no identificó las mismas relaciones que el análisis comparativo de grupos mediante la Prueba T de Student. En futuras indagaciones que permitan ampliar la muestra de CPC se podrán efectuar otros análisis que permitan testear el carácter predictor de las variables reportadas en esta etapa del estudio.

\section{Referencias}

Brussino, Silvina; Patricia Sorribas; Hugo Rabbia y Débora Imhoff (2013) "Enfrentando los desafíos en la evaluación de la participación política: aportes a la discusión sobre indicadores y escalas". Polis 35: 1-17.

De Souza Santos, Boaventura (2003) Democratizar a democracia. Os caminhos da democracia participativa. Porto: Ediçoes Afrontamento.

Eberhardt, María Laura (2010) "Participación política 'directa' en las democracias modernas 'indirectas': Incorporación y desempeño de los mecanismos 'semi-directos' en la ciudad de Buenos Aires (1996-2008)". Disertación para la obtención del grado de doctor en Ciencia Política, Escuela de Política y Gobierno. Universidad de San Martin (Unsam).

Ferrer, Mariona; Lucía Medina y Mariano Torcal (2006) "La participación política: factores explicativos". En José Ramón Montero, Joan Font y Mariano Torcal (eds.) Ciudadanos, asociaciones y participación en España. Madrid: CIS, 133-156. 
Garay Reyna, Zenaida; Inés Ksiazenicki y Patricia Sorribas (2011) "La fragilización de los espacios de participación ciudadana. Un estudio preliminar de las Juntas de Participación Vecinal de Córdoba Capital". Ponencia presentada en el X Congreso Nacional de Ciencia Política de la SAAP, Córdoba, 27 al 30 de julio.

Goldfrank, Benjamin (2007) "¿De la ciudad a la nación? La democracia participativa y la izquierda latinoamericana". Nueva Sociedad 212: 53-66.

Guimond, Serge; Michaël Dambrun; Nicolas Michinov y Sandra Duarte (2003) "Does social dominance generate prejudice? Integrating individual and contextual determinants of intergroup cognitions". Journal of Personality and Social Psychology 84(4): 697-721.

Held, David (1993) Modelos de democracia. Madrid: Alianza.

Imbrasaitë, Jūratė (2009) "Types of participants in political acts: the case of Lithuania". Trabajo presentado en el XXI IPSA World Congress of Political Science, Julio, Santiago - Chile.

Jackman, Robert y Ross Miller (1995) "Voter turnout in the industrial democracies during the 1980s". Comparative Political Studies 27(4): 467-492.

Klesner, Joseph (2007) "Social capital and political participation in Latin America. Evidence from Argentina, Chile, Mexico and Peru". Latin American Research Review 42(2): 1-32.

Lander, Edgardo (1998) "Límites actuales del potencial democratizador de la esfera pública no estatal", en Luis Bresser Pereira y Nuria Cunill Grau (coord.). Lo público no estatal en la reforma del Estado. Buenos Aires: Paidós, CLAD.

Maiz, Ramón (2001) Democracia Participativa. Repensar la democracia como radicalización de la política. Metapolítica 5(8): 72- 95.

Mannarini, Terri; Mónica Legittimo y Cosimo Talò (2008) "Determinants of social and political participation among youth. A preliminary study". Psicología Política 36: 95-117.

Red Ciudadana Nuestra Córdoba (2011, 2012, 2013) Indicadores Ciudadanos Nuestra Córdoba. Disponible en: http://www. nuestracordoba.org.ar.

Rendón Corona, Armando (2004) "Los retos de la democracia participativa". Sociológica 19(54): 183-211. 
Sorribas, Patricia Mariel (2014) "La participación política. Dimensionalidad, amplitud, nivel de involucramiento y factores predictivos. Aportes desde la Psicología Política al desarrollo de la democracia participativa". En Sergio Ilari, Patricia Sorribas y Yanina Guthmann (eds.), III Premio a la innovación y mejoramiento de las políticas públicas: Categoría doctorado. Buenos Aires: Instituto Nacional de la Administración Pública - INAP, 45-97.

Sorribas, Patricia y Silvina Brussino (2013a) "La participación política contenciosa: desarrollo de un modelo explicativo desde la cognición social". Quaderns de Psicologia 15(2): 7-22.

(2013b) "Participación Política Orientada al Sistema Representativo. Dimensiones y factores explicativos". Psicología Política 47: 91-112.

Sorribas, Patricia y Zenaida Garay (2014) "La participación, entre la democracia participativa y la democracia directa. Aportes desde un enfoque psicosocial". Polis Investigación y análisis sociopolítico y psicosocial 10(2): 39-70.

Svampa, Maristella (2005) La sociedad excluyente. Argentina bajo el signo del neoliberalismo. Buenos Aires: Taurus.

Torcal, Mariano; José Montero y Jan Teorell (2006) "La participación política en España: modos y niveles en perspectiva comparada". En José Montero, Joan Font y Mariano Torcal (eds.), Ciudadanos, asociaciones y participación en España. Madrid: CIS, 47-75.

Van Laar, Colette y Jim Sidanius (2001) "Social status and the academic achievement gap: A social dominance perspective". Social Psychology of Education 4(3-4): 235-258.

Wollman, Neill y Robin Stouder (1991) "Believed efficacy and political activity: A test of the specificity hypothesis". Journal of Social Psychology 131 (4): 557-567. 